\title{
REPRESENTATIVENESS OF EUROPEAN BIOCHAR RESEARCH: PART II - POT AND LABORATORY STUDIES
}

\author{
Ruben SAKRABANI ${ }^{\mathrm{a}}$, Jürgen KERN ${ }^{\mathrm{b}}$, Utra MANKASINGH' ${ }^{\mathrm{c}}$, Costanza ZAVALLONI', \\ Giulia ZANCHETTIN ${ }^{\mathrm{e}}$, Ana Catarina BASTOS ${ }^{\mathrm{f}}$, Priit TAMMEORG ${ }^{\mathrm{g}}$, Simon JEFFERY ${ }^{\mathrm{h}}$, \\ Bruno GLASER ${ }^{i}$, Frank G. A. VERHEIJEN ${ }^{j}$ \\ ${ }^{a}$ Cranfield Soil and Agrifood Institute, School of Water, Energy and Environment, Cranfield University, \\ MK43 OAL United Kingdom \\ ${ }^{b}$ Leibniz Institute for Agricultural Engineering and Bioeconomy, Max-Eyth Allee 100, 14469 Potsdam, Germany \\ 'Department of Life and Environmental Sciences, University of Iceland, Sturlugata 7, Reykjavik 107, Iceland \\ ${ }^{d}$ California State University Stanislaus, Agricultural Studies Program, One University Circle, Turlock, CA 95382, USA \\ ${ }^{e}$ University of Udine, Ecosystems Ecology, Via delle Scienze 206, Udine, 33100, Italy \\ ${ }^{f}$ Department of Biology and Centre for Environmental and Marine Studies (CESAM), \\ University of Aveiro, 3810-193 Aveiro, Portugal \\ ${ }^{g}$ Department of Agricultural Sciences, P.O. Box 27 (Latokartanonkaari 5, Plant Production Sciences), \\ FIN-00014 University of Helsinki, Finland \\ ${ }^{h}$ Crop and Environment Sciences Department, Harper Adams University, Newport, Shropshire, \\ TF10 8NB, United Kingdom \\ ${ }^{i}$ Institute of Agronomy and Nutritional Sciences, Martin Luther University Halle-Wittenberg, \\ von-Seckendorff-Platz 3, 06120 Halle/Saale, Germany \\ ${ }^{j}$ Department of Environment and Planning and Centre for Environmental and Marine Studies (CESAM), \\ University of Aveiro, 3810-193 Aveiro, Portugal
}

Submitted 22 Dec. 2016; accepted 12 May 2017

\begin{abstract}
Biochar research is extensive and there are many pot and laboratory studies carried out in Europe to investigate the mechanistic understanding that govern its impact on soil processes. A survey was conducted in order to find out how representative these studies under controlled experimental conditions are of actual environmental conditions in Europe and biomass availability and conversion technologies. The survey consisted of various key questions related to types of soil and biochar used, experimental conditions and effects of biochar additions on soil chemical, biological and physical properties. This representativeness study showed that soil texture and soil organic carbon contents used by researchers are well reflected in the current biochar research in Europe (through comparison with published literature), but less so for soil $\mathrm{pH}$ and soil type. This study provides scope for future work to complement existing research findings, avoiding unnecessary repetitions and highlighting existing research gaps.
\end{abstract}

Keywords: biochar, soil, Europe, pot experiments, laboratory scale, representativeness.

\section{Introduction}

Biochar is a black carbon solid produced by pyrolysis of organic materials (Lehmann et al. 2006) that is often distinguished from charcoal by its intended use as soil amendment (Sohi et al. 2009). During pyrolysis of biomass, carbon (C) atoms form polycondensed aromatic moieties that slow down biological decomposition (Downie et al. 2009). This recalcitrance confers long-term stability of biochar in soil, potentially lasting for centuries (Kuzyakov et al. 2014; Lehmann, Joseph 2015).

Corresponding author: Ruben Sakrabani

E-mail: r.sakrabani@cranfield.ac.uk 
There has been increasing interest in the potential that biochar has for enhancing $\mathrm{C}$ sequestration and improving soil fertility (Spokas, Reikosky 2009), restoring degraded lands (Beesley, Marmiroli 2011), reducing greenhouse gas (GHG) emissions in agriculture (Cayuela et al. 2013; Harter et al. 2014), influencing soil moisture retention (Ulyett et al. 2014) and soil biological activity (Lehmann, Joseph 2015). Recently, Zhang et al. (2016) reviewed 798 publications to provide a quantitative assessment of field and laboratory based experiments from across the globe. They found that the majority of biochars used were produced using kiln or lab batch scale rather than commercial scale pyrolysis, using primarily wood and municipal waste feedstock and that more studies involved laboratory and greenhouse pot experiments rather than field studies. Further, in a recent meta-analyses reported that yield response are dependent on crop type and soil/climatic conditions with more positive effects in the tropics (Jeffery et al. 2011, 2017).

Previous research activities and presentations of results have been uncoordinated, providing a general picture on the topic but with little in-depth investigation of key themes (Tammeorg et al. 2017, this issue). This was one of the stimuli for the initiation of the COST Action TD1107 "Biochar as Option for Sustainable Resource Management" funded by the European Union to bring together key scientists and technologists working in this area. The COST Action TD1107 was a network that aimed to improve data sharing and communication between scientists and stakeholders that developed into a highly useful platform supporting biochar research in Europe. As one of the main tasks of this COST Action was to minimise unnecessary repetition in biochar research, a representativeness survey of European biochar research was conducted consisting of both field-scale (Verheijen et al. 2017, this issue) and pot and laboratory scale studies carried out in Europe. Particularly in the last two years (i.e. 2013-2015), many European researchers joined the COST Action, voluntarily submitting data on their work to a database created to gather information about the type of research and field of application, providing meta-data concerning experimental set up and so producing quantitative, and some qualitative information concerning their studies. This analysis does not qualify for a meta-analysis but it aims to investigate how representative experimental conditions were within biochar research of the COST Action members, regarding biochar properties and environmental application, i.e. feedstock type, pyrolysis conditions, soils, land use and land management. The utility of a representativeness survey is that it gathers and combines multiple aspects of biochar research, providing an overview compared with the published dataset. Such comparison can aid identification of aspects that have not been considered or covered within Europe, so that the missing knowledge gaps can be addressed with further research.

This representativeness survey collates the information from various researchers to provide an overview of their activities and clusters the key outcomes of their research in order to direct further work. The representativeness survey is divided into two categories - part I - field trials (Verheijen et al. 2017, this issue) and part II - pot experiments (the current paper). As such, representativeness analysis is a valuable tool to provide a general overview that is currently missing for biochar studies in Europe.

The objective of this paper is to identify and quantify the observations of biochar effects in pot and laboratory studies and assess how representative the experimental conditions are compared to likely European scale conditions..

\section{Materials and methods}

The information provided by the Representativeness Survey was obtained by using an online questionnaire (http:// webform.cost.european-biochar.org/) formulated by consulting researchers from COST Action TD1107 countries to prioritise key information. The survey was sent to researchers working with biochar in pots, lysimeters and incubation studies and covered aspects related to methods of the experiment, substrates used, processes analysed, type of biochar used and the effects that were observed. As such, the questionnaire was divided into sections that required data related to type of soil used, biochar type feedstock and pyrolysis technology, and effect of biochar on various soil physical, chemical and biological properties. Following submission by respondents within the EU COST Action on a voluntary basis, results from the database were downloaded as a Microsoft Office Excel spreadsheet.

The database was divided into four main parts: (i) General; (ii) Experimental setup; (iii) Biochar treatments and properties; (iv) Measured effects. The first step was to summarise all the sections of the MS Office Excel document to have a visual starting point to facilitate analysis. All entries were grouped following the categories offered by the survey, to obtain a schematic view about the experiments, such as country where the experiment took place, type of experiment, description of soil, description of biochar applied and responses observed. Data for each category were explored by calculating mean, standard deviation, minimum and maximum values using MS Office Excel. Representativeness was deduced from the graphs produced after the data synthesis by comparing with published literature. This consists of infographics, pie and column charts, depending on the type of data considered with the aim of providing a clear visualisation of the 
trends in the experiments. The final step was the interpretation of data considering the present status of knowledge on biochar.

\section{Results}

\subsection{Data collection}

A total of 20 countries contributed to the Representativeness Survey, which resulted in 356 web form submissions. A respondent who submits an online form containing multiple sets of information categorised into four parts as explained above is considered to submit one submission. Table 1 shows the number of submissions for the various countries involved in the COST Action. The submissions were not uniformly distributed with almost half (149) from Spain, which contributed mostly with experiments focusing on ecotoxicological impact, followed by Belgium (37) and Germany (33).

Table 1. Number of submissions per country involved in the Representiveness Survey

\begin{tabular}{lclc}
\hline Austria & 2 & Poland & 5 \\
\hline Belgium & 37 & Portugal & 19 \\
\hline Denmark & 13 & Russia & 8 \\
\hline Estonia & 2 & Slovakia & 9 \\
\hline Finland & 19 & Spain & 149 \\
\hline France & 11 & Sweden & 3 \\
\hline Germany & 33 & Turkey & 2 \\
\hline Greece & 1 & United Kingdom & 5 \\
\hline Italy & 19 & Czech Republic & 1 \\
\hline Netherlands & 4 & Norway & 14 \\
\hline
\end{tabular}

Total $=356$

The climatic zone represented in current biochar research is mainly dependent on the distribution and contribution of different countries: according to the Köppen classification, most of the observations come from experiments under Mediterranean climate (150 submissions), maritime temperate climate (60 submissions) and humid continental climate (31 submissions). This survey did not seek for specific questions about environmental conditions (e.g. annual average temperature, relative humidity and precipitation), the climatic zone only refers to the country where the experiment took place since it was all carried out under controlled conditions.

\subsection{Experimental setup}

A quarter of the experiments were conducted as incubation $(25.0 \%)$ or greenhouse scale $(20.5 \%)$ experiments, with $43 \%$ carried out in climate chambers (Fig. 1). No experiments performed on undisturbed soil cores were submitted.

\subsection{Feedstock for production of biochars}

The feedstocks used for biochar production were grouped into three main categories: (i) lignocellulosic (including woody); (ii) herbaceous (crop residue, green waste, silage); (iii) biosolids (manure, sewage, liquid organic waste). Almost $70 \%$ of the biochar was derived from lignocellulosic feedstock. Biochar produced from herbaceous feedstock accounted for $16 \%$ and biosolids for $9 \%$ of feedstock (Fig. 2).

\subsection{Biochar processing conditions}

The main parameters that are generally modified during char production are carbonisation temperature, type of feedstock and residence time in the heating unit. Changes in these variables influence the proportions of the end products of pyrolysis (biochar, biooil, gas, water) and quality of char produced.

Considering the type of thermochemical conversion of biomass to char, slow pyrolysis covered more than half (64\%) of the experiments, while $17 \%$ of the biochar used in the experiment came from fast pyrolysis. (Fig. 3). The remaining 19\% refers to other methods of heating as gasification, stove, retort kiln and hydrothermal carbonisation (HTC), which are separated from biochars due to their different composition (Schimmelpfennig, Glaser 2012). Nevertheless, these products are defined here as chars. The preference for the slow pyrolysis method, due

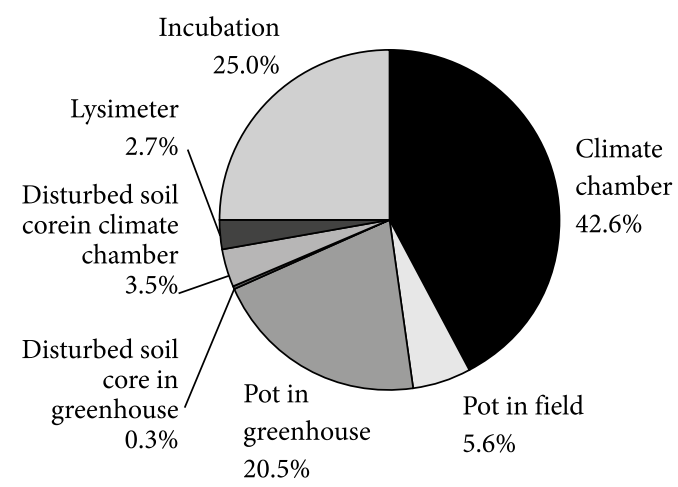

Fig. 1. Survey response of types of experiment $(n=356)$

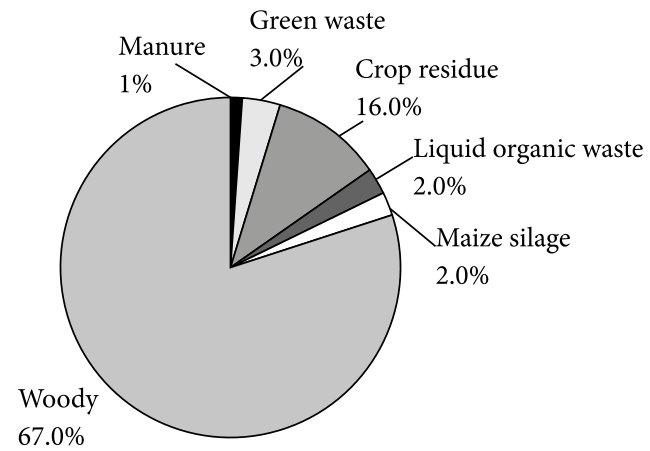

Fig. 2. Survey response of biochar feedstock types $(n=370)$ 


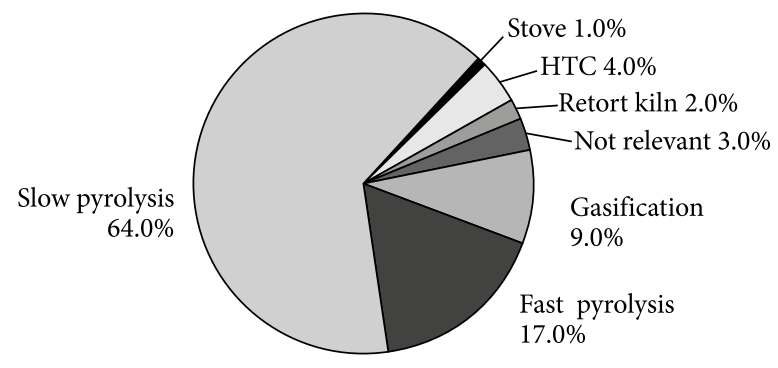

Fig. 3. Survey response of biochar producing technologies $(\mathrm{n}=362)$, HTC - hydrothermal carbonization

to the maximisation of the portion of char produced, reflects the purpose of using biochar as soil amendment and as a possible tool for climate change mitigation (Lehmann, Joseph 2015).

The comparison between the type of feedstock with the temperature of pyrolysis is useful to show how the two aspects, in combination with residence time, are strictly correlated in determining biochar physical and chemical properties.

Biochar was described by the carbonization temperature and residence time, as well as by the method of pretreatment (when applicable) and of its application to pots. Mainly high temperatures $\left(>500^{\circ} \mathrm{C}\right)$ were used for biochar production, covering almost $75 \%$ of the reported experiments (Fig. 4). Most of the carbonization processes (63\%) occurred between 500 and $700{ }^{\circ} \mathrm{C}$, while $11 \%$ took place at over $700{ }^{\circ} \mathrm{C}$. The retention time was generally less than an hour (61\%), but longer periods of carbonization were also present (Fig. 5).

\subsection{Soil type, texture and length of experiments}

All soil types shown in Figure 6 were represented by at least one entry. The presence of Calcisols, Fluvisols and Luvisols was dominant. Calcisols and Fluvisols were mostly associated with responses from Spain indicating dominance of this response. The frequency of these fertile soil types is reflected in the soil texture class, which was mostly loamy (77\%) and details of the breakdown is shown in Figure 7. Calcisols, Fluvisols and Luvisols cover 5\%, 5\% and 6\% of Europe (Soil Atlas of Europe 2005). According to the Soil Atlas Map of Europe, Albeluvisol is the most common soil in Europe (15\%), whilst Podzol is a dominant soil of the northern latitude (14\%). The Cambisols, Chernozems and Leptosols cover 12\%, 9\% and 9\% respectively of European soil. Albeluvisols, Chernozems, and Leptosols were not represented in our database. This shows that in this pot study the representativeness is not well covered in comparison to dominant soil types in Europe.

Soil organic carbon (SOC) content was between 1 and $2 \%$ in about half of reported studies (52\%); $31 \%$ of studies had SOC less than $1 \%$ whilst $17 \%$ had more than

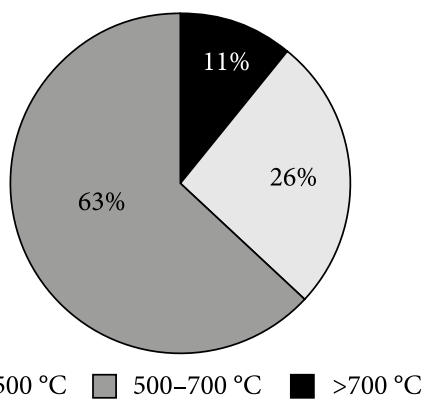

Fig. 4. Survey response of pyrolysis temperature $(\mathrm{n}=328)$

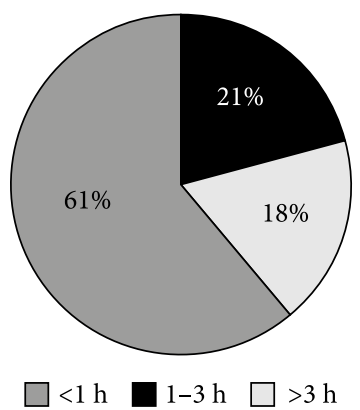

Fig. 5. Survey response of residence time in pyrolysis reactor $(\mathrm{n}=203)$ application

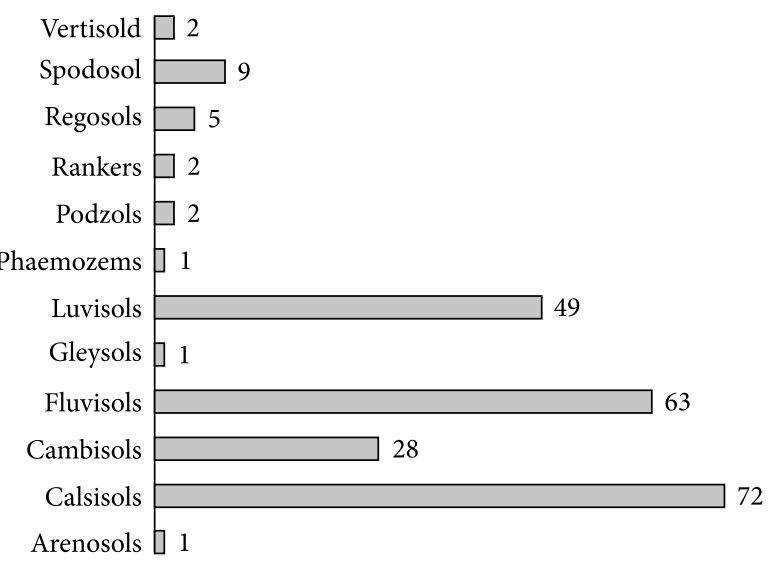

Fig. 6. Survey response of soil types used in biochar application

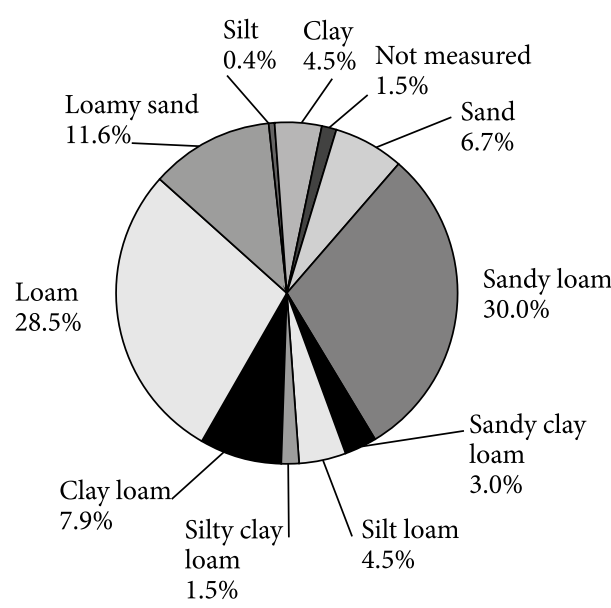

Fig. 7. Survey response of soil texture classes $(n=267)$ 
$2 \%$. Therefore, SOC content was reasonably representative of actual SOC contents in European cropland soils, which mostly have SOC contents between 1-2\% (Toth et al. 2013). The overrepresentation of smaller SOC contents in the laboratory studies is most likely a consequence of the interest to improve soils with low quality, and therefore low SOC contents. Soil $\mathrm{pH}$ was represented by mostly alkaline and neutral soils, while acidic soils accounted for $15 \%$ of studies.

Biochars had an even distribution of $\mathrm{pH}$ and ash content. $60 \%$ of biochar samples contained less than $10 \%$ ash. Seventy percent of all biochars had a pH between 8 and 10 in line with the findings by Verheijen et al. (2017, this issue).

The length of the experiments (i.e. the incubation period) was measured in days. Most of the experiments (53\%) lasted one month or less, while $20 \%$ lasted more than three months. In terms of the incubation temperature, $64 \%$ of the experiments were carried out above $20^{\circ} \mathrm{C}$ (data not shown).

\section{Discussion}

Data were collected using an online survey as this allowed the maximum reach for obtaining data across a range of European Countries. However, such an approach is not without its weaknesses. For example, the timing at which the survey arrived with some institutes may have been more favourable than others in terms of having the time and data availability to allow contribution. Furthermore, the questions posed and format of the questionnaire may have been inhibitory to providing data from some groups than others. These potential biases should be borne in mind while interpreting these data. Nevertheless, 356 web form submissions from 20 countries is a reasonable achievement given the resources and time that was allocated to this task.

The representativeness was judged by comparing the survey response with published literature such as Tammeorg et al. (2017, this issue), Toth et al. (2013) and Zhang et al. (2016) which were used as bench mark in addition to other peer reviewed manuscripts on biochar.

While 20 countries reported on biochar research at the pot scale of experimentation, there is a potential bias driven by the large number of pot scale studies conducted in Spain. However, the majority of these entries were ecotoxicological studies carried out in climate-controlled chambers. As such, the conditions reported from these studies are not "Mediterranean" per se, but representative of standardized conditions based on ISO or OECD guidelines for ecotoxicological assessment of soil and soil materials. In addition to this during a COST Action meeting, biochar effects on soil toxicology was identified as a knowledge gap which encouraged these large submissions.
The authors of this manuscript did not want to remove these submissions in order not to add any bias on the representativeness of this study.

The majority of north-western cropland soil in Europe is acidic; soil in the Mediterranean is generally alkaline. In the partly arid sub-continental zone and temperate mountainous regions, neutral to alkaline soils dominate croplands, and these types of soils are present in considerable areas of the sub-oceanic regions as well (Toth et al. 2013). So the fact that $41 \%$ and $44 \%$ respectively of pot studies used soils from $\mathrm{pH}$ range 6-8 and 8 shows that pot studies are only partly representative.

Around $45 \%$ of the mineral soils in Europe have low or very low organic carbon contents $(0-2 \%)$ and $45 \%$ have a medium content (2-6\%) (Louwagie et al. 2009). Low contents are particularly evident in cropland soils and in southern Europe where $74 \%$ of the land is covered by soils that have less than $2 \%$ of organic carbon in the topsoil $(0-30 \mathrm{~cm})$ (Zdruli et al. 2004). However, areas of low organic carbon can be found almost everywhere, including in cropland soils of northern countries such as Belgium, France, Germany, Norway and the United Kingdom. This study is representative of SOC contents of mineral soils in Europe. However, more than $50 \%$ of EU soil organic carbon stocks are in peatlands (Schils et al. 2008). This study does not list any study using peatland soils, and therefore is not representative of organic soils in Europe. Biochar experiments with a range of peatland soils are therefore recommended to increase European representativeness. A first example has been reported by Kern et al. (2017) in the context of blending peat with biochars in growing media.

Figure 6 shows that Fluvisols and Calcisols are dominant in this study but upon detailed evaluation, these are associated with responses of the online survey from Spain which has skewed the representativeness. However when the information on soil types from Spanish submissions were ignored, the dominant ones are Cambisols and Luvisols. This resonates well with the field scale representativeness reported by Verheijen et al. (2017, this issue).

Uzoma et al. (2011) concluded that the biochar prepared at higher temperature $\left(500{ }^{\circ} \mathrm{C}\right)$ had a greater effect on movement of water in sandy soil as compared to biochar made at lower temperatures $\left(300\right.$ and $\left.400{ }^{\circ} \mathrm{C}\right)$. In that experiment, it was found that the pyrolysis temperature and the mixing rate of biochar both affected the hydraulic properties of sandy soil. Existing research (Kinney et al. 2012; Ulyett et al. 2014; Ahmed et al. 2016) shows that most of the biochar has been applied to course textured soils and its positive effect on soil physical aspects is reflected in this representativeness survey (Fig. 7).

A section of the online survey also contained questions related to effects of biochar on various soil physical, chemical and biological properties which can be mapped 
to the thematic areas described by Tammeorg et al. (2017, this issue). Since the questions posed on the effects were categorised as "positive", "negative" or "no effect" which was deemed as too subjective, further discussions about this results are not elaborated here. Nevertheless it has led to postulation of research questions which can be useful to fulfil knowledge gaps identified by Tammeorg et al. (2016). Amongst the aspects that needs further work are as follows:

1. What are the effects of low temperature biochar on soil properties?

This survey indicates that the smallest percentage of biochar used in this study are produced using temperature $<500{ }^{\circ} \mathrm{C}$. It will be very valuable if further investigation is carried out using biochar produced at low temperatures. At lower temperatures stability of the carbon in biochar is reduced which can possibly facilitate easier use as substrate for soil microbial proliferation. This can provide new insights where the tradeoff is between carbon sequestration (associated with more biochar produced using high temperature) and carbon mineralisation and lability (associated with biochar produced using low temperatures).

2. What are the effects of livestock manure and biosolids based biochar on soil properties?

This survey shows that woody and crop residue based biochar are dominant as shown in Figure 2. It will be very valuable to explore alternative sources as feedstock for biochar especially biosolids which has usually high contents of phosphorus and how mineralisable this will be in soil to meet crop demands.

3. What are the effects of biochar on undisturbed soil cores?

In this survey, biochar has been added to pots or incubation studies where it is mixed and then the effects studied. However the effects on undisturbed soil cores have not been studied so far. This can entail using biochar of different particle sizes that can be added as top dressing to the undisturbed soil cores.

4. What are the effects of biochar on common soil types in the EU?

The Soil Atlas Map of Europe, shows that Albeluvisol is the most common soil in Europe (15\%), whilst Podzol is a dominant soil of the northern latitude (14\%). This survey shows that the dominant soils types in these studies are not Albeluvisol or Podzol indicating that further work is needed in this aspect.

The above list of questions is non-exhaustive and only demonstrates the opportunity that this Representativeness Survey can lend itself to. Whilst this Representativeness Survey in itself may not represent all biochar research in the EU, it allows opportunity for future work through knowledge gap analysis.

Since this representativeness survey was focused on providing an overview of the extent of current biochar research in Europe, there are some limitations in this approach. Due to the overwhelming response from Spain, this has potentially skewed the representativeness of this survey. Whilst this has to be acknowledged, one benefit is that this survey has revealed that the responses from Spain are associated with research related to ecotoxicology. This can provide opportunities for collaboration amongst EU COST Actions Members. Whilst this manuscript was targeting to showcase representativeness of biochar research in the EU associated with pots and laboratory scale studies, in reality this was not fully achieved due to lack of response to the online survey. The lack of response is something that we can only postulate as being a combination of, data not being ready for further analysis, unwillingness to participate or survey may seems too onerous despite that the explanation that the expectation is not for the respondent to fill every box but as many as possible and where relevant. It was also challenging to convince colleagues to submit data, which have not been published before and to show that further publishing of original data is still possible after using for the online survey.

This survey did depend on number of respondents so where regions got a good response it enabled to better showcase research from that part of the EU. However, where response was poor from certain regions of the EU, it just shows that response was poor for this survey and does not fully reflect that research is missing in this region. This is a limitation of this survey.

\section{Conclusions}

This study provides an overview of generic responses from adding biochar to soil in laboratory scale experiments and can be used as an indicative tool but should not be over-interpreted. The data presented here show that currently the regions in the European continent are not well represented. Further to this, there is a moderate representation of soil $\mathrm{pH}$ and weaker representation of soil type, but good representation in terms of SOC content and soil texture in mineral soils. This representiveness survey can also be used to link with the field scale experiment to corroborate any observations that can provide some seed corn ideas that can be further developed into future projects. In terms of the effects of biochar on the Thematic Areas, many aspects of the results were in line with published work and confirmed what was known about biochar until present time. The novelty of this work lies in the approach adopted to provide an overview on soil responses observed when applied with biochar in non-field conditions and as well as starting point for future research on this topic has been brought to light. 


\section{Acknowledgements}

The authors are grateful for the EU COST Action TD1107 (Biochar as option for sustainable resource management) for funding the meetings that helped to enable this work. We also wish to acknowledge the Portuguese Foundation for Science and Technology (FCT) for the fellowships of A.C. Bastos (SFRH/BPD/98231/2013) and F.G.A. Verheijen (SFRH/BPD/107913/2015), the financial support to CESAM [UID/AMB/50017/2013], to FCT/ MEC through national funds and the co-funding by FEDER, within PT2020 Partnership Agreement and Compete 2020. The authors are very indebted to Jan Sorensen who was extremely helpful in converting the Excel version of the survey into an online version that could be easily edited, stored and used to retrieve information. Johannes Harter was also very instrumental in retrieving information for the online version of the survey and collating it into a format that authors could use for this publication. We would like to thank all contributors from the various Member States as without your information this paper would not have been feasible.

\section{References}

Ahmed, A.; Kurian, J.; Raghavan, V. 2016. Biochar influences on agricultural soils, crop production, and the environment: a review, Environmental Reviews 24(4): 495-502.

https://doi.org/10.1139/er-2016-0008

Beesley, L.; Marmiroli, M. 2011. The immobilisation and retention of soluble arsenic, cadmium and zinc by biochar, Environmental Pollution 159: 474-480.

https://doi.org/10.1016/j.envpol.2010.10.016

Cayuela, M. L.; Sánchez-Monedero, M. A.; Roig, A.; Hanley, K.; Enders, A; Lehmann, J. 2013. Biochar and denitrification in soils: when, how much and why does biochar reduce $\mathrm{N}_{2} \mathrm{O}$ emissions?, Scientific Reports 3. Article No. 1732.

https://doi.org/10.1038/srep01732

Downie, A.; Crosky, A.; Munroe, P. 2009. Physical properties of biochar, in J. Lehmann, S. Joseph (Eds.). Biochar for environmental management: science and technology. London, UK: Earthscan, 13-32.

Harter, J.; Krause, H-M.; Schuetter, S.; Ruser, R.; Fromme, M.; Scholten, T.; Kappler, A.; Behrens, S. 2014. Linking $\mathrm{N}_{2} \mathrm{O}$ emissions from biochar-amended soil to the structure and function of the N-cycling microbial community, International Society for Microbial Ecology Journal 8(3): 660-674. https://doi.org/10.1038/ismej.2013.160

Jeffery, S.; Abalos, D.; Prodana, M.; Bastos, A. C.; van Groenigen, J. W.; Hungate, B.; Verheijen, F. G. A. 2017. Biochar boosts tropical but not temperate yields, Environmental Research Letters 12(5).

https://doi.org/10.1088/1748-9326/aa67bd

Jeffery, S.; Verheijen, F. G. A.; Van Der Velde, M.; Bastos, A. C. 2011. A quantitative review of the effects of biochar application to soils on crop productivity using meta-analysis, Agriculture, Ecosystems \& Environment 144(1): 175-187. https://doi.org/10.1016/j.agee.2011.08.015
Kern, J.; Tammeorg, P.; Shanskiy, M.; Sakrabani, R.; Knicker, H.; Kammann, C.; Tuhkanen, E.-M.; Smidt, G.; Prasad, M.; Tiilikkala, K.; Sohi, S.; Gasco, G.; Steiner, C.; Glaser, B. 2017. Synergistic use of peat and charred material in growing media - an option to reduce the pressure on peatlands?, Journal of Environmental Engineering and Landscape Management (this issue).

Kinney, T. J.; Masiello, C. A.; Dugan, B.; Hockaday, W. C.; Dean, M. R.; Zygourakis, K. 2012. Hydrologic properties of biochars produced at different temperatures, Biomass Bioenergy 41: 34-43. https://doi.org/10.1016/j.biombioe.2012.01.033

Kuzyakov, Y.; Bogomolova. I.; Glaser, B. 2014. Biochar stability in soil: decomposition during eight years and transformation as assessed by compound-specific ${ }^{14} \mathrm{C}$ analysis, Soil Biology and Biochemistry 70: 229-236. https://doi.org/10.1016/j.soilbio.2013.12.021

Lehmann, J.; Joseph, S. 2015. Biochar for environmental management. London and New York: Routledge.

Lehmann, J.; Gaunt, J.; Rondon, M. 2006. Bio-char sequestration in terrestrial ecosystems - a review, Mitigation \& Adaptation Strategies for Global Change 11: 395-419. https://doi.org/10.1007/s11027-005-9006-5

Louwagie, G.; Gay, S. H.; Burrell, A. 2009. Final report on the project 'Sustainable Agriculture and Soil Conservation (SoCo)' JRC Scientific and Technical Reports. European Commission, Joint Research Centre, Luxembourg.

Schils, R.; Kuikman, P.; Liski, J.; van Oijen, M.; Smith, P.; Webb, J.; Alm, J.; Somogyi, Z.; van den Akker, J.; Billett, M.; Emmett, B.; Evan, C.; Lindner, M.; Palosuo, T.; Bellamy, P.; Jandl, R.; Hiederer, R. 2008. Final report on review of existing information on the interrelations between soil and climate change (Climsoil). Final Report for the European Commission's Directorate-General for the Environment. European Commission, Brussels.

Schimmelpfennig, S.; Glaser, B. 2012. One step forward toward characterization: some important material properties to distinguish biochars, Journal of Environmental Quality 41: 10011013. https://doi.org/10.2134/jeq2011.0146

Sohi, S.; Lopez-capel, E.; Krull, E; Bol, R. 2009. Biochar, climate change and soil a review to guide future research. CSIRO, Glen Osmond.

Soil Atlas of Europe. 2005. European Soil Bureau Network, European Commission. Office for Official Publications of the European Communities, Luxembourg. 128 p.

Spokas, K. A.; Reikosky, D. C. 2009. Impacts of sixteen different biochars on soil greenhouse gas production, Annals of Environmental Science 3: 179-193.

Tammeorg, P.; Bastos, A. C.; Jeffery, S.; Rees, F.; Kern, J.; Graber, E.; Ventura, M.; Kibblewhite, M.; Amaro, A.; Budai, A.; Cordovil, C. M. d. S.; Domene, X.; Gardin, G.; Gasco, G.; Horak, J.; Kammann, C.; Kondrlova, E.; Laird, D.; Loureiro, S.; Martins, M. A. S.; Panzacchi, P.; Prasad, M.; Prodana, M.; Puga, A. P.; Ruysschaert, G.; Sas-Paszt, L.; Silva, F. C.; Teixeira, W. G.; Tonon, G.; Vedove, G. D.; Zavalloni, C.; Glaser, B.; Verheijen, F. G. A. 2017. Biochars in soils: towards the required level of scientific understanding, Journal of Environmental Engineering and Landscape Management (this issue). https://doi.org/10.3846/16486897.2016.1239582

Tóth, G.; Jones, A.; Montanarella, L. 2013. The LUCAS topsoil database and derived information on the regional variability of cropland topsoil properties in the European Union, 
Environmental Monitoring and Assessment 185(9): 74097425. https://doi.org/10.1007/s10661-013-3109-3

Ulyett, J.; Sakrabani, R.; Hann, M.; Kibblewhite, M. 2014. Impact of biochar addition on water retention, nitrification and carbon dioxide evolution from two landy loam soils, European Journal of Soil Science (Special Issue on Biochar) 65(1): 96-104. https://doi.org/10.1111/ejss.12081

Uzoma, K. C.; Inoue, M.; Andry, H.; Fujimaki, H.; Zahoor, A.; Nishihara, E. 2011. Effect of cow manure biochar on maize productivity under sandy soil condition, Soil Use Manage 27: 205-212. https://doi.org/10.1111/j.1475-2743.2011.00340.x

Verheijen, F. G. A.; Mankasingh, U.; Penizek, V.; Panzacchi, P.; Glaser, B.; Jeffery, S.; Bastos, A. C.; Harter, J.; Tammeorg, P.; Kern, J.; Zavalloni, C.; Zanchettin, G.; Sakrabani, R. 2017.
Representativeness of European biochar research: Part I field experiments, Journal of Environmental Engineering and Landscape Management (this issue).

Zdruli, P.; Jones, R. J. A.; Montanarella, L. 2004. Organic matter in the soils of Southern Europe European soil bureau technical report. Publications Office of the European Union, Luxembourg.

Zhang, D.; Yan, M.; Niu, Y.; Liu, X.; van Zwieten, L.; Chen, D.; Bian, R.; Cheng, K.; Li, L.; Joseph, S.; Zheng, J.; Zhang, X.; Zheng, J.; Crowley, D.; Fillet, T. R.; Pan, G. 2016. Is current biochar research addressing global soil constraints for sustainable agriculture?, Agriculture, Ecosystems and Environment 226: 25-32.

https://doi.org/10.1016/j.agee.2016.04.010

Ruben SAKRABANI. PhD, Senior Lecturer in Soil Chemistry, Cranfield Soil and Agrifood Institute, Cranfield University, 30 peer-reviewed articles. H-index 12 (Google Scholar).

Jürgen KERN. PhD, Senior Scientist of Biogeochemistry, Leibniz Institute for Agricultural Engineering and Bioeconomy, Germany, 1211 citations. H-index 16 (Google Scholar).

Utra MANKASINGH. PhD, Research Fellow in the Faculty of Life and Environmental Sciences at the University of Iceland. 6 publications in international peer reviewed journals, 3 major reports, 159 citation; H-index 4 (Google Scholar).

Costanza ZAVALLONI. PhD, Assistant Professor in plant science in the Agricultural Studies program, California State University Stanislaus, Turlock, 19 peer-reviewed articles in international scientific journals, 1 book chapter, 514 citations, H-index 11 (Google Scholar).

Giulia ZANCHETTIN. MSc graduate student in Ecology and Soil Science and was funded under the Short Term Scientific Mission (within the Biochar COST Action) to analyse representativeness datasets.

Ana Catarina BASTOS. PhD, research fellow of the Portuguese Science Foundation (FCT), at Department of Biology and Centre for Environmental and Marine Studies (CESAM), University of Aveiro, Portugal. 22 publications in international peer-reviewed journals, 1 European Commission report, 4 book chapters. 1745 citations; H-index 13 (Google Scholar).

Priit TAMMEORG. DSc, Postdoctoral Researcher in Agroecology, Dept of Agricultural Sciences, University of Helsinki, 11 peer-reviewed articles in international scientific journals, H-index 6 (TR WoS).

Simon JEFFERY. PhD, Senior Lecturer at Harper Adams University, Department of Crop and Environment Sciences, 2031 citations, H-index 16 (Google Scholar).

Bruno GLASER. PhD, Professor of Soil Biogeochemistry, Institute of Agronomy and Nutritional Sciences, Martin Luther University Halle-Wittenberg, Germany, 140 peer-reviewed articles in scientific journals. H-index 39 (WoS).

Frank G. A. VERHEIJEN. PhD, research fellow of the Portuguese Science Foundation (FCT), Centre for Environmental and Marine Studies (CESAM), University of Aveiro, Portugal. 25 publications in international peer-reviewed journals, 7 major European reports, 5 book chapters. 2348 citations; H-index 16 (Google Scholar). 